\title{
MANUFACTURING A PERSONALISED COMPOSITE MATERIAL DRIVER'S SEAT
}

\author{
Paweł Żur, Alicja Kołodziej, Adam Nowak, Andrzej Baier
}

Silesian University of Technology, Department of Mechanical Engineerning, Konarskiego 18A, 44-100 Gliwice, Poland

Corresponding author: Alicja Kołodziej, alicko196@gmail.com

\begin{abstract}
The paper presents the process of 3D-printing and laminating a composite material driver's seat for Silesian Greenpower's electric vehicle. Silesian Greenpower is a student's interfaculty project, of which aim is to design, build and race a small electric race car. Aerodynamics and lightweight construction play a major role, so each element applied in the car is optimized and customized to fit in the vehicle. For this purpose, 3D-printing technology is often used by the team. A seat model made on the basis of the driver's anthropometric model was presented in the paper. Subsequently, after the appropriate adaptation of the obtained surfaces to interconnect them with each other, the FDM technology was applied using PET-G material. To increase the strength of the connections of the printed elements and to strengthen the design and stability of the chair, the element was laminated using carbon fibre and epoxy resin. The lamination process is presented and discussed in the article.
\end{abstract}

Key words: 3D-printing, anthropometry, carbon fibre, composite, electric vehicle, laminate

\section{INTRODUCTION}

\subsection{Silesian Greenpower}

Silesian Greenpower is a students' project of which aim is to design and build an electric race car. There are two main components of the car which are designed in Siemens NX software: the chassis and the carbon-fiber bodywork. Shape and dimensions of some elements of the chassis (shown in Figure 1) such as roll bar are specified by the regulations of Greenpower formula races [3].

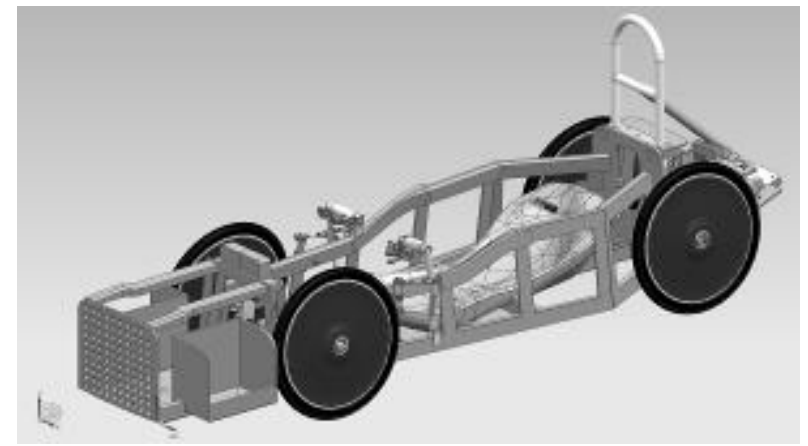

Fig. 1. The chassis of the new Silesian Greenpower team's vehicle
The shape of the body is unrestricted and each of the teams designs it on their own.

In this particular case, aerodynamic drag represents 70 percent of all the frictions that are the consequences of car motion. Each of the electric cars taking part in Greenpower races is equipped with the same electric motor. The motor is powered by two $12 \mathrm{~V}$ batteries with a capacity of $36 \mathrm{Ah}$ each. The main goal is to drive as many laps as possible in a certain amount of time [2].

Based on the results obtained in the analysis of new solutions, constructions, and parts are being applied in the vehicle. Each year adjustments have to been made in order to make a car compliant with new regulations [15].

\section{MATERIALS AND METHODS}

\subsection{D Scanning}

3D laser scanning describes the three-dimensional measurement of the surface of an object by analyzing the reflected light of a laser beam that is scanned over the surface of the object. Polar coordinates with origin in the scanning system are determined by angle and range measurements. If the reflected light intensity is also recorded, an image of an object is recorded, in which each measured 3D point is assigned an intensity value depending on the reflectance of the object surface against the wavelength of the scanning laser. Initially, laser scanners were developed for aeronautical applications where laser scanning of the ground from an airplane creates a digital terrain model. These scanners are increasingly used in short-range applications, starting as weld inspection tools and extending the range from capturing building interiors to measuring complex structures (terrestrial laser scanning, TLS). [8] 3D scanning is based on a triangulation mechanism. Triangulation works on the basic trigonometric principle - a point or a laser line is projected onto an object from a handheld device, and the sensor measures the distance to the surface. The location of the scanner can be determined using 
reference elements on the surface to be scanned or using an external tracking method. External tracking often takes the form of a laser tracker (to determine the position of the sensor) with an integrated camera or a photogrammetric solution using 3 or more cameras providing the full six degrees of freedom of the scanner.

Both techniques typically use infrared emitting diodes connected to the scanner, which are visible to the camera (s) through filters to ensure immunity to ambient light.

The Kinect 3D scanner uses the same technology a mid-range 3D Scanner might have, a camera, and an infrared camera used to calculate the field of depth of and around an object. The device has many advantages over existing 3D scanning technologies: it can measure depth and color at the same time, which makes it very suitable for scanning objects quickly. The depth sensor does not interfere with the scene in the visual spectrum. Finally, its use is no different from a video camera; end-users deal with it intuitively and are able to collect data with just a few minutes of training. [16]

Kinect, however, has a low resolution so it can be difficult to capture small details of the image, it is recommended to use it for the overall shape of the human body scan. Kinect 3D will get the best results if it works with a scanning head travel speed of about $10-20 \mathrm{~mm} / \mathrm{sec}$. [16]

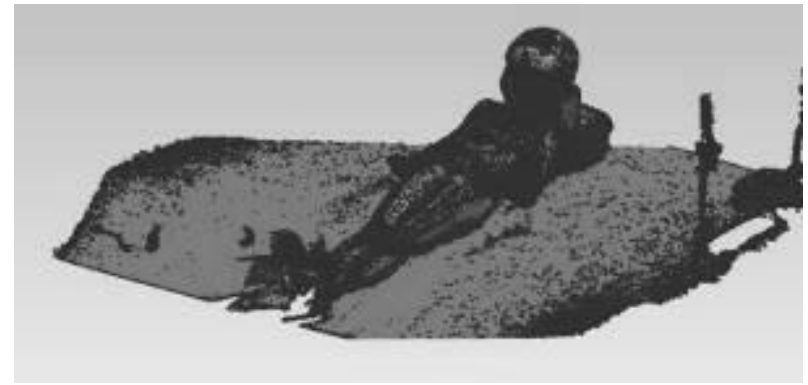

Fig. 2. Raw scan of the driver. [16]

Figure 2 shows an example scan made with the Kinect camera with ReconstructMe software.

\subsection{Technology used: FDM}

Additive manufacturing is relatively new technology which has been invented in the 1980s and developed dynamically in last two decades $[3,11]$.

All 3D printing processes offer advantages and disadvantages. The type of 3D printer chosen for an application often depends on the materials to be used and how the layers in the finished product are bonded. 3D Printing is a member of a group of layer manufacturing techniques which have the primary distinguishing feature of creating parts by the controlled addition (rather than subtraction) of material [11]. The three most commonly used 3D printer technologies are: selective laser sintering (SLS), thermal inkjet (TIJ) printing, and fused deposition modeling (FDM) [6].

FDM technology is the most popular manufacturing technology used on the market. In FDM thermoplastic material is pressed through the nozzle to form the modeled shape. The 3D printer used to fabricate test specimens was made by 3DGence and had a $0.5 \mathrm{~mm}$ nozzle. Some parts of the Silesian Greenpower vehicles are 3D-printed - e.g. wheel fairing and mirror casing. This method allows customizing the shape of an element and its manufacturing [3].

\subsection{Materials used: PLA, PET-G}

PLA - Polylactic acid is proving to be a viable alternative to petrochemical-based plastics for many applications. PLA is biodegradable and bioactive thermoplastic polyester. It can be made by fermentation of starch-containing agricultural products like potato (waste), rice and alike. The degradation time is 18 to 24 months. PLA copolymer is a blend with glycolide/lactide ratio of $85 / 15$ which has a moderate crystallinity and a degradation rate [4].

Polyethylene Terephthalate (PET) is the most widely used plastic in the world. It is used in applications such as the creation of water bottles, right up to the fibers of clothing. Most foods and beverages are delivered and packaged using PET.

When it comes to 3D printing, PETG material is the particular version of this plastic that is used in 3D printing processes whilst the $G$ is standing for "glycol-modified". This is added during polymerization to the material composition.

PLA filament is characterized by high level of strength and low moisture absorption. It is naturally transparent and reduces environmental impact by biodegradation [9]. PET-G filament is well known for its high printing speed, better thermal resistivity, minimal schrinkage and being odorless while printing. Both materials are characterized by low warping effect. Comparison of mechanical and printing properties of 3D-printing filaments manufactured by BigRep has been presented in Table 1.

Table 1. Mechanical properties of PLA and PET-G filaments $[9,10]$

\begin{tabular}{|c|c|c|}
\hline Property & PLA & PET-G \\
\hline Density $\left[\mathrm{g} / \mathrm{cm}^{3}\right]$ & 1.24 & 1.27 \\
\hline Tensile Stregth [MPa] & 60 & 50 \\
\hline Flexural Modulus [GPa] & 3.80 & 2.145 \\
\hline $\begin{array}{l}\text { Charpy Impact, notched } \\
{\left[\mathrm{J} / \mathrm{cm}^{2}\right]}\end{array}$ & 0.750 & 0.720 \\
\hline $\begin{array}{l}\text { Viscat Softening Point } \\
{\left[{ }^{\circ} \mathrm{C}\right]}\end{array}$ & 60 & 70 \\
\hline Heating Bed $\left[{ }^{\circ} \mathrm{C}\right]$ & 60 & 80 \\
\hline $\begin{array}{l}\text { Print Temperature } \\
\text { Guideline }\left[{ }^{\circ} \mathbf{C}\right]\end{array}$ & 205 & $25-220$ \\
\hline
\end{tabular}




\subsection{Laminated composites}

Laminate composites are orthotropic, consisting of multiple layers of oriented or randomly oriented fibres bound together by a matrix in which each layer of fibres has a two-dimensional (ie, planar) orientation. Laminated composite materials are used extensively in the mechanical and aerospace industries, especially for the fabrication of high performance structures. As wellknown, there are many methods for manufacturing laminated composite components and different manufacturing or curing processes may yield different mechanical properties of the components $[1,14]$.

Currently, MDF material is used to make a mold that will be used to make a laminated composite. This material is milled to reflect the geometry of the surface that will be laminated. The mold is then coated with sealing agents for micropores present in the material and with anti-adhesive agents to ease the removal of the finished composite from the mold without damaging it. The mold milling process is cost effective only for the production of molds that will be used repeatedly.

This paper id focused on an innovative approach to the production of molds for making laminated composites - molds made by 3D printing in FDM technology, which is much more profitable in unit production and does not require highly specialized equipment like a CNC milling machine.

\subsection{Vacuum infusion method}

The applied lamination technology was vacuum infusion molding process which is becoming increasingly popular for the production of large composite parts. The Vacuum Infusion Process is a technique that uses vacuum pressure to drive resin into a laminate. Materials are laid dry into the mold and the vacuum is applied before resin is introduced. Once a complete vacuum is achieved, resin is literally sucked into the laminate via carefully placed tubing (Figure 3 ). This process is aided by an assortment of supplies and materials [13, 5]. In a typical hand lay-up, reinforcements are laid into a mold and manually wet out using brushes, rollers, or through other means. An improvement on that method is to use a vacuum bag to suck excess resin out of the laminate. Vacuum bagging greatly improves the fiber-to-resin ratio, and results in a stronger and lighter product [13].

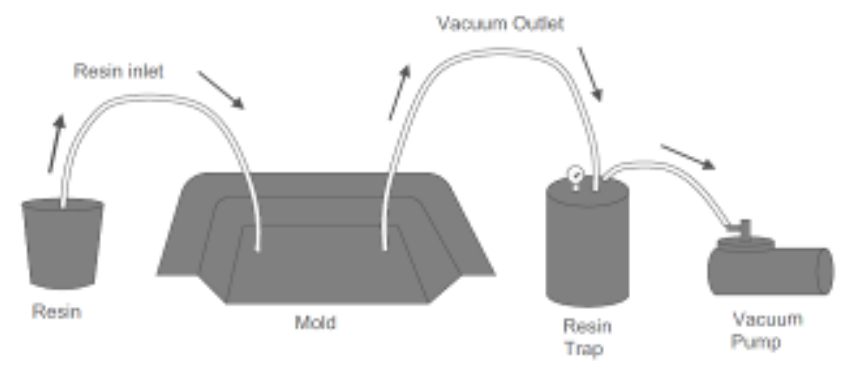

Fig. 3. Scheme of infusion method [13]
Vacuum infusion provides a number of improvements over traditionally vacuum bagged parts. These benefits include: better fiber-to-resin ratio, less wasted resin, very consistent resin usage, unlimited set-up time, cleaner process.

Though set-up is time-limit free, it is somewhat more complicated. Vacuum bagging requires the placement of only the vacuum tubing. Vacuum infusion requires not only vacuum tubes but resin inlets as well, not to mention in-bag extensions of these tubes. Placement of these vacuum and resin lines varies from part to part, and there is no one way to set them up [13].

\subsection{Scope of the research}

The aim of this article was to make a seat from a composite material. In the first phase of research, a test model with smaller dimensions and a simpler shape than the target chair was prepared. For this purpose, the helmet fairing of an electric car driver was used (Figure 4). The mold of the fairing was printed out of PLA material. The lamination process highlighted the defects of the material used. The fairing was deformed because the temperature of polymerization turned out to be too high in relation to the strength properties of the material used. For further research, the material was changed to PET-G, which is characterized by a higher temperature at which the material deforms.

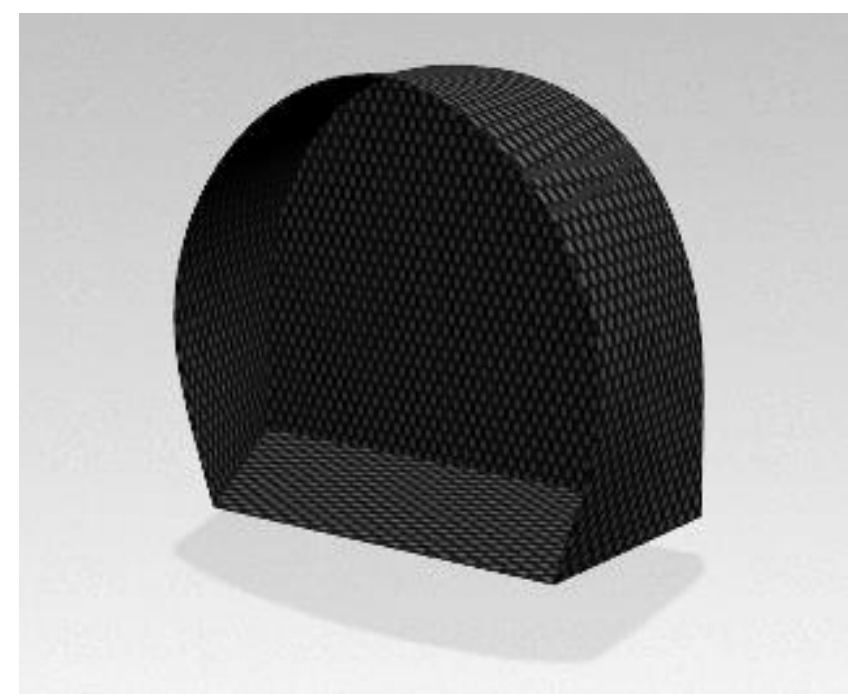

Fig. 4. Helmet fairing

\subsection{Driver anthropometric model}

The seat was aligned to the car and driver. A 3D scan and a CAD model of the car were used. Due to the scanning technology used and the way the driver was positioned, a scan of the driver's back was not possible. In order to properly fit the seat to the car, several key points were taken from the scan as shown in the Figure 5. 


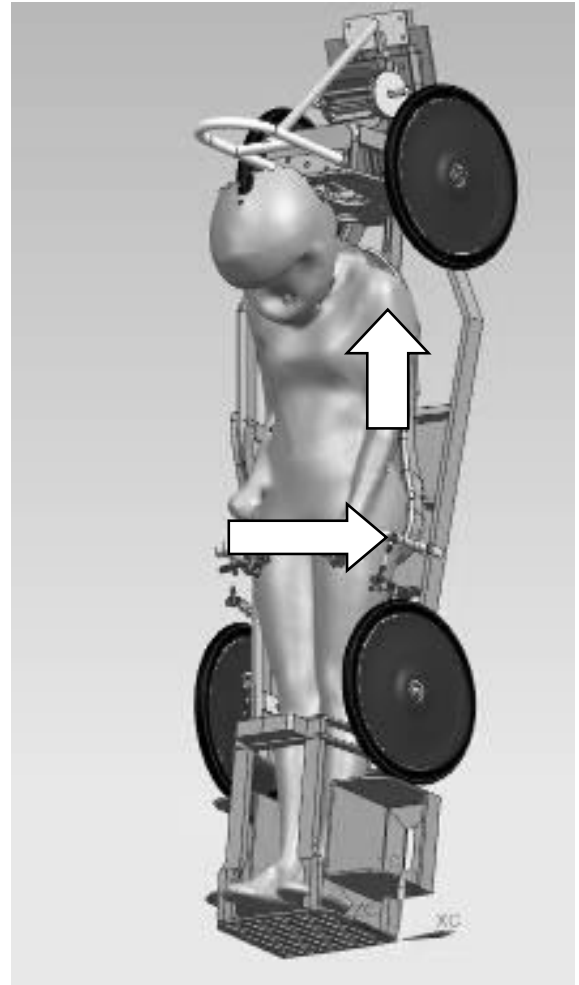

Fig. 5. Scan of the driver in the car

Using these reference points, the correct position has been established and the shape of the seat has been adapted to the driver, which allowed going to the next stage.

\subsection{Printing and assembling the mold}

When the alignment process was completed, the mold of the seat was prepared. In order to avoid costly and time-consuming milling of the mold, it was decided to use the FDM printing method. This method allows to make even complex and complicated shapes in a fast and relatively cheap way. The mold was printed from 8 parts, which were connected with each other by means of designed grooves and also by means of glue.

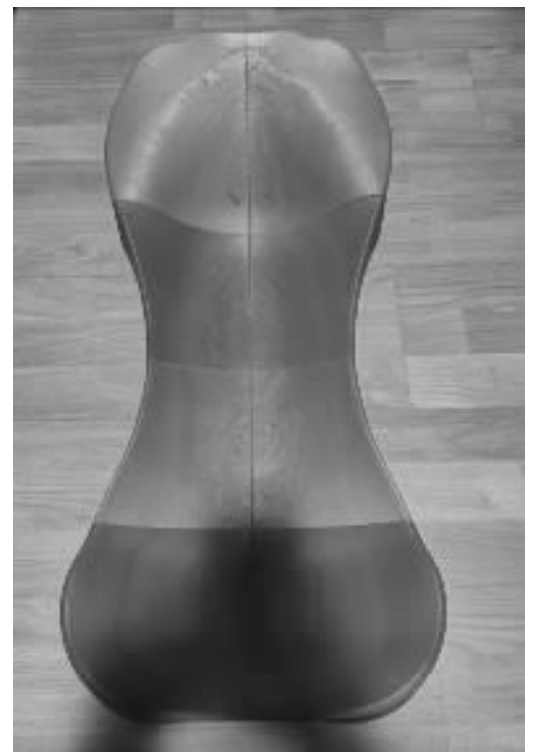

Fig. 6. Printed mold
The mold shown in Figure 6 has been coated with release agents and separation wax to avoid problems with separating the finished product from the mold.

\subsection{Vacuum infusion process}

In the first phase a smaller test model has been laminated using infusion method - a helmet fairing.

The first step of the vacuum infusion process was sealing the mold. The mold has been covered with three thin layers of sealing agent to reduce micropores present in the mold, and three thin layers of anti-adhesive agent to ease the removal of the finished composite driver's seat from the mold. Additional layer of a mold release wax has been applied on the edges of the mold.

Then three layers of $200 \mathrm{~g}$ glass fibre cloth have been placed on the mold. The strengthening phase - glass fibre - has been covered with peel ply material for easier release of the finished laminate. Then the infusion mesh has been fitted as a top layer. The infusion mesh is used for an even distribution of a resin during the infusion process. Additional spiral tubing is applied to allow an even flow of the resin in more difficult to reach areas of the mold.

Then the mold was placed in a vacuum bag sealed around the edges to provide tightness needed for vacuum. The bag had one inlet with a clamp and one outlet (Figure 7). The direction of the flow of the resin has been marked on figure below.

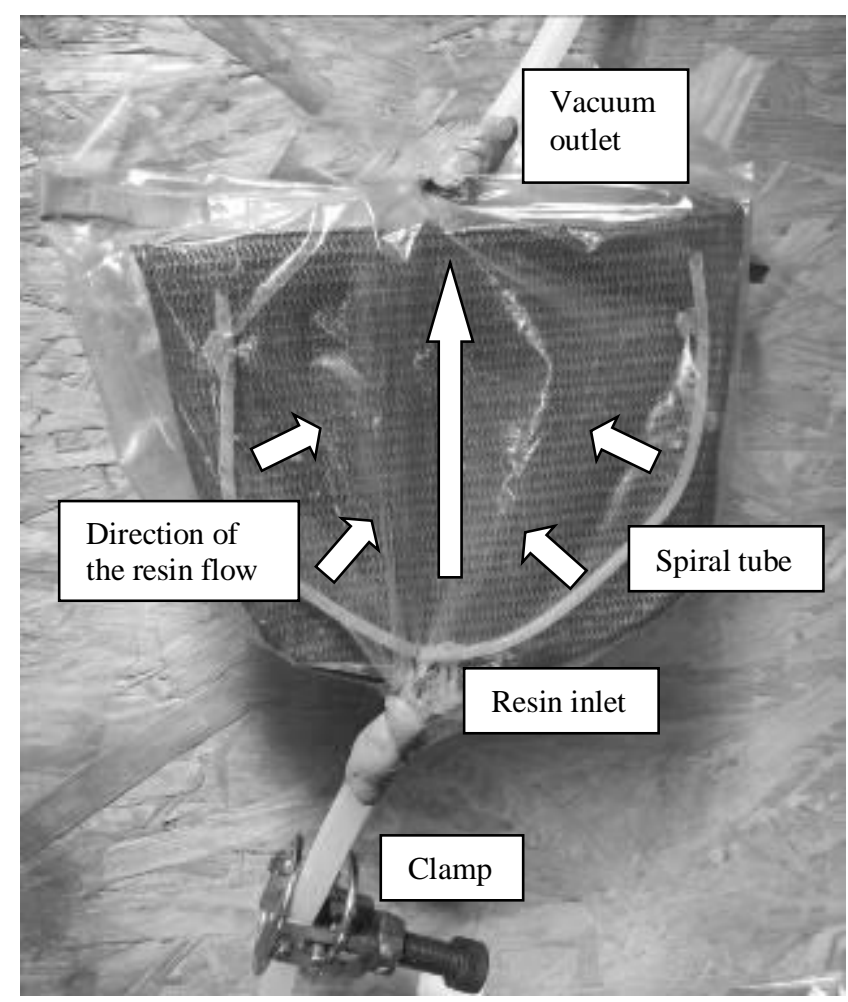

Fig. 7. Vacuum bag used in resin infusion method

The amount of the resin needed for the infusion process has been selected on the basis of the Table 2 . 
Table 2. Resin consumption [ml] in infusion [7]

\begin{tabular}{|c|c|c|c|c|c|}
\hline & \multicolumn{4}{|c|}{ Number of plys of $200 \mathrm{~g}$ cloth } \\
\hline & & 1 & 2 & 3 & 4 \\
\hline \multirow{7}{*}{$\begin{array}{c}\text { Area } \\
\text { of } \\
\text { part } \\
\text { in } \\
\text { square } \\
\text { meters }\end{array}$} & 0.1 & 183 & 197 & 210 & 223 \\
\hline & 0.2 & 267 & 293 & 320 & 347 \\
\hline & 0.3 & 350 & 390 & 430 & 470 \\
\hline & 0.5 & 517 & 583 & 650 & 717 \\
\hline & 0.75 & 725 & 825 & 925 & 1025 \\
\hline & 1 & 933 & 1067 & 1200 & 1333 \\
\hline & 1.25 & 1142 & 1308 & 1475 & 1642 \\
\hline
\end{tabular}

The area of the part was $0.25 \mathrm{~m}^{2}$, so the amount of LG700 resin applied was $430 \mathrm{ml}$ of resin and HG700 hardener in ratio 100:30. The vacuum obtained was 0.8 bar. The time needed for the resin to cure was 12 hours. The composite obtained in the process did not show satisfactory properties (Figure 8). Due to high temperature being released during the polymerization process, in this case the polymerization temperature was too high for the printing material used - PLA. As a result of mold melting, the surface quality and the appropriate shape were not maintained. The form of the actual model - i.e. the driver's seat - was printed from PET-G material, which is characterized by a higher temperature resistance than PLA.

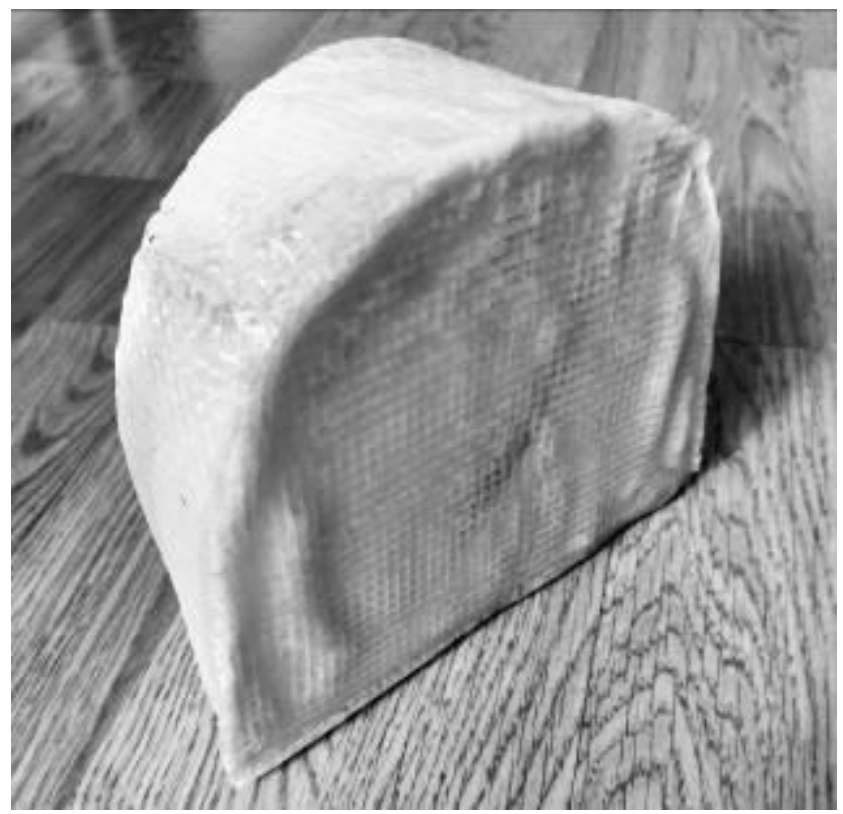

Fig. 8. Laminated test model of a helmet's fairing

Parameters applied for infusion process of the driver's seat were exact same as for the helmet's fairing, although the material applied for the seat was carbon fibre. Also 3 layers have been applied. The area of the part was $0.8 \mathrm{~m}^{2}$, so the amount of LG700 resin applied was $1200 \mathrm{ml}$ of resin and HG700 hardener in ratio 100:30. As a result of the infusion process a laminated carbon fibre composite seat has been manufactured (Figure 9).

\section{RESULTS AND DISCUSSION}

\subsection{Result}

As an end result an ultra-thin and durable electric car driver's seat was obtained.

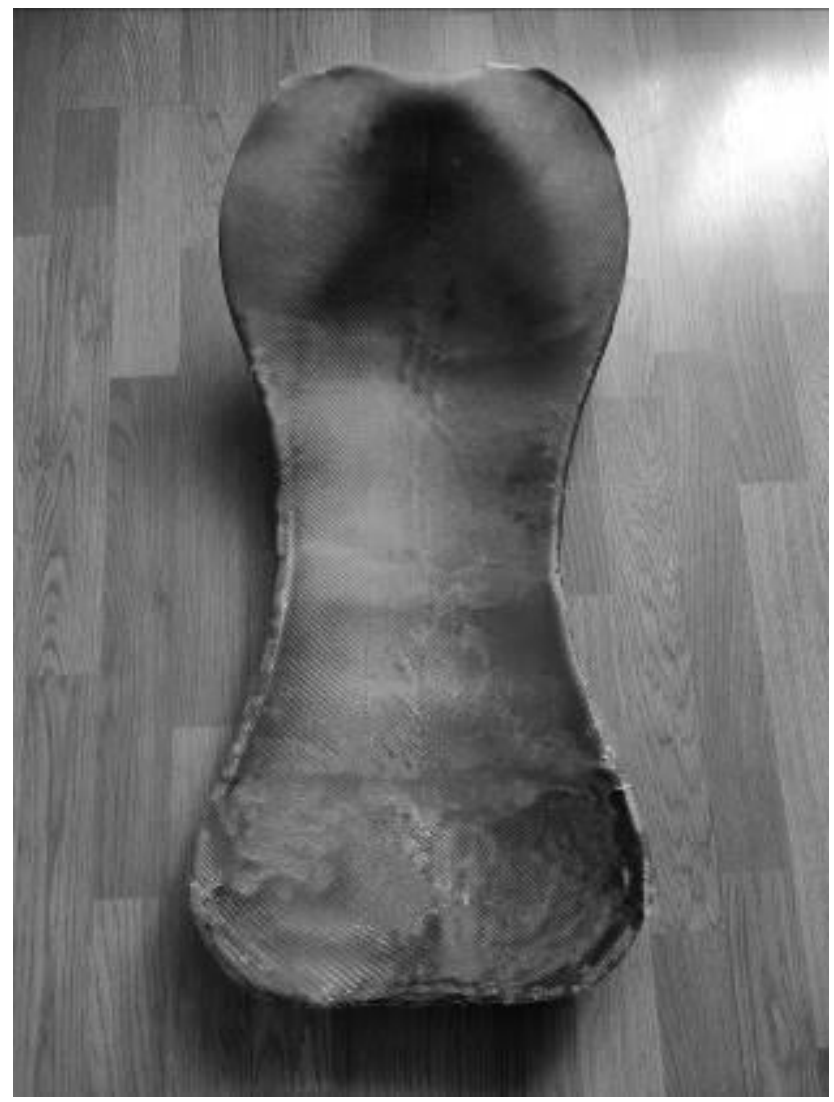

Fig. 9. Seat after removal from the mold

The overall quality of the manufactured part was satisfactory. Change of the mold material from PLA to PET-G had positive influence on the process flow of the resin infusion method. The mold has retained its shape during the polymerization process of the resin.

\subsection{Discussion}

The use of 3D printing in FDM technology enables the production of molds for unit production of laminated composites. Using PLA filament as a mold material does not bring the expected results. This form deformed due to the high polymerization temperature of the resin. Replacing PLA with PET-G eliminated this undesirable effect.

The infusion process, despite the time-consuming preparation and the need for more materials, compared to manual lamination brings better final results. The process is also much cleaner. The quality of the obtained surface (Figure 10) is also better than in the manual process. 


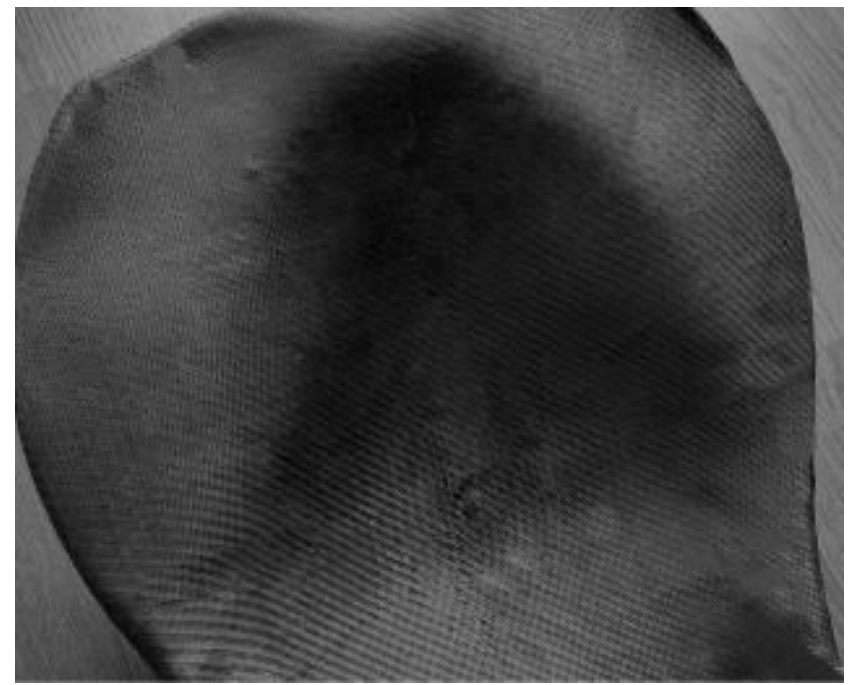

Fig. 10. Surface quality of the laminated seat

This is due to the fact that the material is constantly pressed against the mold by the prevailing vacuum in the bag. The method used allows for multiple modifications of the fabric placed on the mold, which results in its more precise arrangement.

\section{CONCLUSIONS}

The use of 3D printing in FDM technology allows the production of molds for the unit production of laminated composites. The use of 3D printing significantly reduces production costs in relation to milled molds made of e.g. MDF.

The use of PET-G as a material for the mold gives satisfactory results of the quality of the obtained surface and avoids deformation of the mold during resin polymerization.

The proposed production method using the infusion method results in a better quality of the obtained laminate surface and shape reproduction.

The infusion method has a higher process purity than hand laminating. The use of 3D scanning technology allows precise adjustment of the model.

\section{REFERENCES}

1. Abrate, S. (1994). Impact on laminated composites: recent advances.

2. Baier, A., Grabowski, Ł., Stebel, Ł., Komander, M., Konopka, P., Kołodziej, A., \& Żur, P. (2018). Numeric analysis of airflow around the body of the Silesian Greenpower vehicle, In MATEC Web of Conferences, 178, p. 05014, EDP Sciences.

3. Baier, A., Zur, P., Kolodziej, A., Konopka, P., \& Komander, M. (2018). Studies on optimization of 3Dprinted elements applied in Silesian Greenpower vehicle. IOP Conference Series: Materials Science and Engineering $\mathbf{4 0 0} 022010$.

4. Giordano, R. A., Wu, B. M., Borland, S. W., Cima, L. G., Sachs, E. M., \& Cima, M. J. (1997).
Mechanical properties of dense polylactic acid structures fabricated by threedimensional printing. Journal of Biomaterials Science, Polymer Edition, 8(1), 63-75.

5. Hammami, A., \& Gebart, B. R. (2000). Analysis of the vacuum infusion molding process. Polymer composites, 21(1), 28-40.

6. Ligon, S. C., Liska, R., Stampfl, J., Gurr, M., \& Mülhaupt, R. (2017). Polymers for 3D printing and customized additive manufacturing. Chemical reviews, 117(15), 10212-10290.

7. Lipson, H., \& Kurman, M. (2013). Fabricated: The new world of $3 D$ printing. John Wiley \& Sons.

8. Luhmann, T., Robson, S., Kyle, S., \& Boehm, J.

(2013). Close-range photogrammetry and $3 D$ imaging. Walter de Gruyter.

9. Proto3000 - Rapid Prototyping Engineering Solutions - BigRep PLA Filament Material Data Available from: https://www.matweb.com Accessed: 16/04/2020.

10. Proto3000 - Rapid Prototyping Engineering Solutions - BigRep PET-G Filament Material Data Available from https://www.matweb.com Accessed: 16/04/2020.

11.Sachs, E., Cima, M., Cornie, J., Brancazio, D., Bredt, J., Curodeau, A., ... \& Michaels, S. (1993). Three-dimensional printing: the physics and implications of additive manufacturing. CIRP annals, 42(1), 257-260.

12. Vacuum Infusion - Resin Consumption Accessed from: http://www.talkcomposites.com/ Accessed: 16/04/2020.

13. Vacuum Infusion - The Equipment and Process of Resin Infusion Accessed from: http://composites.ugent.be Accessed: 16/04/2020. 14. Wang, W. T., \& Kam, T. Y. (2000). Material characterization of laminated composite plates via static testing. Composite Structures, 50(4), 347-352. 15. Żur, P., Baier, A., \& Kolodziej, A. (2019, April). Influence of Selected Parameters of the Motor Controller on the Current Characteristics of the DC Brush Motor Used in the Silesian Greenpower's Vehicle. In IOP Conference Series: Materials Science and Engineering 520(1), p. 012010). IOP Publishing. 16. Żur, P., Baier, A., \& Kołodziej, A. (2020). Chassis Geometry Optimization based on 3D-scans of the Ergonomic Driving Position. International Journal of Mechanical Engineering and Robotics Research, 9(8).

Received: March 08, 2021 / Accepted: December 20, 2021 / Paper available online: December 25, 2021 (C) International Journal of Modern Manufacturing Technologies 